Internat. J. Math. \& Math. Sci.

Vol. 22, No. 3 (1999) 463-468

S 0161-1712<99>22463-5

(c) Electronic Publishing House

\title{
A PROPER SUBCLASS OF MACLANE'S CLASS $\mathscr{A}$
}

\author{
MAY HAMDAN
}

(Received 26 June 1997 and in revised form 20 April 1998)

\begin{abstract}
The MacLane's class $\mathscr{A}$ of analytic functions is the class of nonconstant analytic functions in the unit disk that have asymptotic values at a dense subset of the unit circle. In this paper, we define a subclass $\mathscr{R}$ of $\mathscr{A}$ consisting of those functions that have asymptotic values at a dense subset of the unit circle reached along rectifiable asymptotic paths. We also show that the class $\mathscr{R}$ is a proper subclass of $\mathscr{A}$ by constructing a function $f \in \mathscr{A}$ that admits no asymptotic paths of finite length.
\end{abstract}

Keywords and phrases. Analytic functions, asymptotic path, tangential approximation, proper subset, construction, unit disc.

1991 Mathematics Subject Classification. 30A99, 30D40.

1. Preliminaries. In all what follows, $f$ is a nonconstant analytic function on the unit disk.

DEFINITION 1 [2]. We say that a simple curve $\Gamma: z(t), 0 \leq t<1$ is a boundary path ending at $\zeta$ if $|z(t)| \rightarrow 1$ as $t \rightarrow 1$ and if $\bar{\Gamma} \cap C=\{\zeta\}$. The number $a$ is called an asymptotic value associated with $\zeta$ if there is a boundary path $\Gamma$ such that $f(z(t)) \rightarrow a$ as $t \rightarrow 1$ and $\bar{\Gamma} \cap C=\{\zeta\}$. In that case, we call $\Gamma$ an asymptotic path.

DEFinition 2. We define the set $A(f)$ to be the set of all points $\zeta$ at which $f$ has an asymptotic value. In particular, we denote by $A_{a}$ the set of all points $\zeta$ associated with the asymptotic value $a$, and by $A_{\infty}$ the set of all points $\zeta$ associated with the asymptotic value $\infty$. We also define the set $A^{R}$ to be the set of all points on the unit circle at which $f$ has asymptotic values reached along rectifiable asymptotic paths.

DEFINITION 3 [3]. If $A(f)$ is a dense subset of $C$, we say that $f \in \mathscr{A}$, the MacLane class of analytic functions and we define the set $\mathscr{R}$ to be the subset of $\mathscr{A}$ for which $A^{R}$ is a dense subset of the unit circle $C$.

DEFINITION 4 [1]. Let $H \subset D$ be a relatively closed subset of $D$. We say that $H$ is an Arakelyan set or $H \in K(D)$ if, for every $z_{0} \in D-H$, there is a boundary path $\Gamma_{0} \subset D-H$ which connects $z_{0}$ to $C$, that is, if there is a boundary path $\Gamma_{0}: z(t), 0 \leq t<1$, such that $z(t) \in D-H, z(0)=z_{0}$ and $d(z(t), C) \rightarrow 0$ as $t \rightarrow 1$. Here, $d(z(t), C)$ denotes the distance from $z(t)$ to $C$.

DEFINITION 5. Let $H \subset D$. We say that $H$ is a set of tangential approximations (by analytic functions of $H$ ) provided that, for each function $g$ continuous on $H$ and analytic on the interior $H^{0}$ of $H$, and for each positive continuous function $\epsilon(t), 0<$ $t<1$, there is an analytic function $f$ on $D$ such that, for all $z \in H$,

$$
|f(z)-g(z)|<\epsilon(d(z, C)) .
$$


Note that when $H^{0}=\phi$, the function $g$ is only required to be continuous on $H$.

LEMMA 1 [1]. Let $H \in K(D)$, and $H^{0}=\phi$. Then $H$ is a set of tangential approximation. This is Arakelyan's theorem.

2. Main theorem. $\mathscr{R}$ is a proper subset of $\mathscr{A}$.

Proof. The strategy is to construct a function $f \in \mathscr{A}$ by approximating a function $g$ on an Arakelyan set $H$, with $H^{0}=\phi$, using Lemma 1.

The set $H$ is the union of

(a) a sequence of circles converging to the circumference $C$, each having small equally spaced gaps in it, and

(b) the boundary paths that snake through the gaps.

The gaps in a circle have a total length that approaches zero quickly as the circles approach the circumference. Also, the gaps on consecutive circles are rotated enough so that the asymptotic paths approaching a point of the circumference that pass through the circles only in the gaps (of most of the circles) have infinite length.

The set $H$ so constructed turns out to be a set of tangential approximation. We define a continuous function $g$ on the set $H$ as follows. On each circle (minus its gaps), the function is constant, and on consecutive circles, it has values $0,1,2,3,0,1,2,3,0$, $1,2,3, \ldots$. Along the asymptotic paths of the set $H$, the function approaches infinity (uniformly) as the modulus approaches 1 . Arakelyan's theorem allows us to extend the function $g$ into an analytic function $f$ with the desired property. The set $H$ and the function $g$ and, consequently, $f$ are constructed in such a way that any asymptotic path along which $f$ converges must be either funneled only through the gaps of the circle (if the limit is other than $0,1,2$, or 3 ), or must eventually only hit at most one of four circles (hence, pass through the gaps most of the time). In either cases, the asymptotic path must be of infinite length because it is trying to avoid the circles minus the gaps on which the function keeps alternating between the four finite values. More specifically, we start with a sequence of circles $\left\{C_{n}^{j}\right\}$ converging to the circumference $C$ each having small equally spaced gaps so that the circle minus the gaps form the first set of $\operatorname{arcs}\left\{\gamma_{n, k}^{j}\right\},(n=2,3, \ldots, k=1,2, \ldots, n, j=0,1,2,3)$. The $\operatorname{arcs}\left\{\gamma_{n, k}^{j}\right\}$ will be positioned so that if any asymptotic path were to avoid a 'good number' of them, that asymptotic path would have to be of infinite length. The second set of arcs $\left\{\Gamma_{p \backslash q}\right\},(q=1,2,3, \ldots$ and $p=1, \ldots, q-1)$ consists of boundary paths ending at a dense subset of $C$. The set $H$ is the union of the sets $\left\{\gamma_{n, k}^{j}\right\}$ and $\left\{\Gamma_{p \backslash q}\right\}$. First, we show that $H \in K(D)$ then we define a function $g$ on $H$ with the property that $g \rightarrow \infty$ as $|z| \rightarrow 1$ along the boundary paths $\Gamma_{p \backslash q}$, while the function $g$ takes four different constant values on subarcs $\left\{\gamma_{n, k}^{j}\right\}$ of four consecutive concentric circles $C_{n}^{j}$, $j=0,1,2$, 3. Finally, we show that $g$ and $H$ satisfy the conditions of Lemma 1 and that, for an appropriate choice of $\epsilon(t)>0$, the function $f$ in the conclusion of the lemma has the desired property: $f \in \mathscr{A}-\mathscr{R}$.

CONSTRUCTION OF THE SET $H$.

$$
H=\left(\cup_{j=0}^{3} \cup_{n=1}^{\infty} \cup_{k=1}^{n}\left(\gamma_{n, k}^{j}\right)\right) \cup\left(\cup_{q=1}^{\infty} \cup_{p=1}^{q-1}\left(\Gamma_{p \backslash q}\right)\right)
$$


We only take the values of $p$ and $q$ that are relatively prime $((p, q)=1)$.

(1) Description of the $\operatorname{arcs} \gamma_{n, k}^{j}, n=2,3, \ldots ; j=0,1,2,3 ; k=1,2, \ldots, n$. We start with a sequence of circles $\left\{C_{n}^{0}\right\}, n=2,3, \ldots$, centered at the origin, each of radius $r_{n}^{0}=1-1 / n$, and we let $D_{n}, n=2,3, \ldots$ be the annulus $D_{n}=\left\{r_{n}^{0} \leq|z|<r_{n+1}^{0}\right\}$. Let $D_{1}$ be the disc $\left\{z:|z|<r_{2}^{0}\right\}$. Within each annulus $D_{n}$, we define three concentric circles $C_{n}^{j},(j=0,1,2,3)$ centered at the origin, of radius $r_{n}^{j}=r_{n}^{0}+(j /(4 n(n+1)))$, where $j=1,2,3$. So, the three circles are equally spaced consecutively between $C_{n}^{0}$ and $C_{n+1}^{0}$. Note that here and in all that follows, $j$ is used as an index rather than an exponent in $r_{n}^{j}$. On every circle $C_{n}^{j},(n=2,3, \ldots ; j=0,1,2,3)$ we arrange $n$ (equally spaced) arcs $\left\{\gamma_{n, k}^{j}\right\},(k=1,2, \ldots, n)$ of equal length so that the gaps

$$
C_{n}^{j}-\cup_{j}\left(\gamma_{n, k}^{j}\right)
$$

consist of $n$ open $\operatorname{arcs} \sigma_{n, k}^{j}$, each of length $\pi r_{n}^{j} / 2^{n}$, such that, for $j=0$ or 2 , the arcs $\sigma_{n, k}^{j}, k=1,2, \ldots, n$, all have their midpoint at the point $r_{n}^{j} e^{2 k \pi i / n}$, whereas for $j=1$ or 3 , the $\operatorname{arcs} \sigma_{n, k}^{j}, k=1,2, \ldots, n$, have their midpoint at $r_{n}^{j} e^{(2 k+1) \pi i / n}$ (a rotation of angle $\pi / n$ from the previous case). The arcs $\sigma_{n, k}^{j}$, which are the gaps on the circles, are rotated enough so that a boundary path funneling through a 'good number' of them would be of infinite length. Note that the length of $\gamma_{n, k}^{j}$ is

$$
\left|\gamma_{n, k}^{j}\right|=\frac{2 \pi r_{n}^{j}}{n}-\frac{\pi r_{n}^{j}}{n}
$$

In the future, we refer to the $\operatorname{arcs} \gamma_{n, k}^{j}\left(\sigma_{n, k}^{j}\right), j=0,1,2,3$, in $D_{n}$ as the arcs $\gamma_{n}\left(\sigma_{n}\right)$ provided there is no ambiguity. The distance from a point of $\sigma_{n}$ on $C_{n}^{j}$ to a point of $\sigma_{n+1}$ on $C_{n+1}^{j}$ is at least

$$
\frac{\pi r_{n}^{j}}{n}-\frac{\pi r_{n}^{j}}{2^{n}} \text { for } j=0,1,2
$$

because of the arrangement of the $\operatorname{arcs} \gamma_{n}$ and $\sigma_{n}$. Consider a curve $J_{n} \subset \overline{D_{n}}$ such that

$$
J_{n} \cap C_{n}^{j} \neq \phi \quad \text { for } j=0,1,2,3 \text {. }
$$

Suppose, in addition, that $J_{n} \cap \gamma_{n, k}^{j} \neq \phi$ for at most one value of $j \in\{0,1,2,3\}$. Therefore, $J_{n}$ crosses a pair of circles $C_{n}^{j}$ and $C_{n+1}^{j}$ for some value of $j \in\{0,1,2,3\}$ at points of some arcs $\sigma_{n}$ and $\sigma_{n+1}$. By the previous remark, the length of such a path is

$$
\left|J_{n}\right|>\pi r_{n}^{j}\left(\frac{1}{n}-\frac{1}{2^{n}}\right) \text {. }
$$

(By doing so, we have made sure that the gaps on consecutive circles are rotated enough so that asymptotic paths approaching a point of the circumference that pass through the circles only in the gaps (of 'most' of the circles) have infinite length.)

(2) Description of the boundary paths $\Gamma_{p / q}$. For $q=1,2, \ldots$, and $p=1,2, \ldots, q-1$ and $(p, q)=1$, let

$$
S=\left\{e^{2 \pi i p / q}\right\} .
$$

Note that $S$ is a dense subset of $C$. We define a sequence of disjoint boundary paths $\left\{\Gamma_{p / q}\right\}$, where $p$ and $q$ are as mentioned above, and such that $\Gamma_{p / q} \cap\{z:|z|=r\}$ consists 
of exactly one point for all $r$ satisfying $r_{q}^{0} \leq r<1$. ( $\Gamma_{p / q}$ is not defined for $r<r_{q}^{0}$.) We need to construct the $\operatorname{arcs} \Gamma_{p / q} \cap C_{n}^{j}$ for $j \in\{0,1,2,3\}$, and $n=q, q+1, \ldots$, so that

$$
\Gamma_{p / q} \cap C_{n}^{j} \neq \Gamma_{p^{\prime} / q^{\prime}} \cap C_{n}^{j},
$$

if $p / q \neq p^{\prime} / q^{\prime}$. In the special case of $\Gamma_{1}$, we make

$$
\Gamma_{1} \cap C_{n}^{j}= \begin{cases}r_{n}^{j} e^{\pi i / 2^{n+1}} & \text { for } j \in\{0,2\}, \\ r_{n}^{j} e^{\pi i / 2^{n+1}} e^{\pi i / n} & \text { for } j \in\{1,3\} .\end{cases}
$$

For $q>1$, we make

$$
\Gamma_{p / q} \cap C_{n}^{j}= \begin{cases}r_{n}^{j} e^{(2 \pi i\lfloor n p / q\rfloor / n)+\left(p \pi i / 2^{n+1} q\right)} & \text { for } j \in\{0,2\} \\ r_{n}^{j} e^{(2 \pi i\lfloor p / q\rfloor / n)+\left(p \pi i / 2^{n+1} q\right)} e^{\pi i / n} & \text { for } j \in\{1,3\},\end{cases}
$$

where \lfloor\rfloor is the greatest integer function. The first part of the argument, $2 \pi i\lfloor n p / q\rfloor / n$, is the center of the 'gap' $\sigma_{n, k}^{j}$, while the second part of the argument, $p \pi i / 2^{n+1} q$, determines the distance from the point $\Gamma_{p / q} \cap C_{n}^{j}$ to the midpoint of the arc $\sigma_{n, k}^{j}$; it ensures that the point of intersection is still within $\sigma_{n, k}^{j}$. Such paths $\Gamma_{p / q}$ must end at the dense subset $S$ of $C$ since, for any $p$ and $q$ as described above,

$$
r_{n}^{j} \rightarrow 1 \quad \text { and } \quad e^{(2 \pi i\lfloor n p / q\rfloor / n)+\left(p \pi i / 2^{n+1} q\right)} \longrightarrow e^{2 \pi i p / q} \quad \text { as } n \rightarrow \infty .
$$

Note that two different paths intersect the circle $C_{n}^{0}$ at two different points since if $p / q \neq p^{\prime} / q^{\prime}$, then

$$
r_{n}^{j} e^{(2 \pi i\lfloor n p / q\rfloor / n)+\left(p \pi i / 2^{n+1} q\right)} \neq r_{n}^{j} e^{\left(2 \pi i\left\lfloor n p^{\prime} / q^{\prime}\right\rfloor / n\right)+\left(p^{\prime} \pi i / 2^{n+1} q^{\prime}\right)} .
$$

We define

$$
\Gamma_{p / q}: z(t), \quad 0 \leq t<1
$$

to be the polygonal arc that begins at

$$
z(0)=r_{q}^{0} e^{(2 \pi i p / q)+\left(p \pi i / 2^{q+1} q\right)} .
$$

(Observe that the arc $\Gamma_{p / q}$ starts on the arc $\sigma_{q, p}^{0}$ whose midpoint is of argument $2 \pi i p / q$.) In the annulus $\overline{D_{n}}$, for $n=q, q+1, \ldots$ the $\operatorname{arc}$ joins $r_{n}^{0} e^{(2 \pi i\lfloor n p / q\rfloor / n)+\left(p \pi i / 2^{n+1} q\right)}$ on $C_{n}^{0}$ successively

$$
\begin{aligned}
& \text { to } r_{n}^{1} e^{(2 \pi i\lfloor n p / q\rfloor / n)+\left(p \pi i / 2^{n+1} q\right)} e^{\pi i / n} \text { on } C_{n}^{1} \text {, } \\
& \text { to } r_{n}^{2} e^{(2 \pi i\lfloor n p / q\rfloor / n)+\left(p \pi i / 2^{n+1} q\right)} \text { on } C_{n}^{2} \text {, } \\
& \text { to } r_{n}^{3} e^{(2 \pi i\lfloor n p / q\rfloor / n)+\left(p \pi i / 2^{n+1} q\right)} e^{\pi i / n} \text { on } C_{n}^{3} \text {, and finally } \\
& \text { to } r_{n+1}^{1} e^{(2 \pi i\lfloor n p / q\rfloor / n)+\left(p \pi i / 2^{n+1} q\right)} \text { on } C_{n+1}^{0} \text {. }
\end{aligned}
$$

Observe the rotation provided by the $e^{\pi i / n}$ factor. From the definition of $\Gamma_{p / q}$ and from the fact that $\Gamma_{p / q} \cap C_{n}^{j} \neq \Gamma_{p^{\prime}} / q^{\prime} \cap C_{n}^{j}$ in case $p / q \neq p^{\prime} / q^{\prime}$, the paths are disjoint. As an illustration, we take the path $\Gamma_{4 / 5}$. Its initial point is the point $r_{5}^{0} e^{2 \pi i(4 / 5)+4 \pi i / 2^{6} 6}$ on the $\operatorname{arc} \sigma_{4,5}^{0} \subset C_{5}^{0}$. The path $\Gamma_{4 / 5}$ ends at the point of $C$ of the argument $2 \pi i(4 / 5)$. The second circle that $\Gamma_{4 / 5}$ crosses at a point on an arc $\sigma_{n}$ whose midpoint is of argument 
$2 \pi i(4 / 5)$ (more informally, the gap at $2 \pi i(4 / 5)$ ), is the circle $C_{10}^{0}$. The $k$ th such circle is the circle $C_{5 k}^{0}$. (Note that this is a justification for using the greatest integer function. In fact, the solution for $n$ in the equation $e^{2 \pi i\lfloor n 4 / 5\rfloor / n}=e^{2 \pi i(4 / 5)}$ is $n=5 k$.) Now, consider the paths $\Gamma_{1 / 3}$ and $\Gamma_{1 / 4}$. They both intersect $C_{8}^{0}$ at different points of the same arc $\sigma_{8,2}^{0}$. In fact,

$$
\Gamma_{1 / 3} \cap C_{8}^{0}=r_{8}^{0} e^{(2 \pi i[8(1 / 3)\rfloor / 8)+\left(\pi i / 2^{9} 3\right)}=r_{8}^{0} e^{(\pi i / 2)+\left(\pi i / 2^{9} 3\right)}
$$

while

$$
\Gamma_{1 / 4} \cap C_{8}^{0}=r_{8}^{0} e^{(2 \pi i[8(1 / 4)\rfloor / 8)+\left(\pi i / 2^{9} 3\right)}=r_{8}^{0} e^{(\pi i / 2)+\left(\pi i / 2^{9} 4\right)} .
$$

In general, there might be more than two paths $\Gamma_{p / q}$ intersecting a circle $C_{n}^{0}$ at different points of the same arc $\sigma_{n, k}^{j}$. However, there are finitely many $\left(n^{2}\right)$ such paths since $q \leq n$ and $p<q$. Note that the arcs $\left\{\gamma_{n}\right\}$ and $\left\{\sigma_{n}\right\}$ have been arranged so that any such path $\Gamma_{p / q}$ is of infinite length since, $\Gamma_{p / q} \cap D_{n}$ contains an arc joining a point of $\sigma_{n, k}^{j}$ to a point of $\sigma_{n, t}^{j+1}, j=0,1,2$, similar to the arcs $J_{n}$ in a previous remark. Finally, define the set $H$ to be the following disjoint union:

$$
H=\left(\cup_{j=0}^{3} \cup_{n=1}^{\infty} \cup_{k=1}^{n}\left(\gamma_{n, k}^{j}\right)\right) \cup\left(\cup_{q=1}^{\infty} \cup_{p=1}^{q-1}\left(\Gamma_{p \backslash q}\right)\right) .
$$

The set $H$ is relatively closed because every $\operatorname{arc} \gamma_{n, k}^{j}$ is closed and $\overline{\Gamma_{p \backslash q}}$ are closed arcs in $\overline{D_{n}}$.

Proof of $H \in K(D)$. Let $z_{0} \in D-H$. We need to find a path $\Gamma_{0} \in D-H$ that connects $z_{0}$ to $C$. Note that $z_{0} \notin \gamma_{n}$ for any $n$, and $z_{0} \notin \Gamma_{p / q}$ for any $\Gamma_{p / q} \subset H$. Choose $n$ so that $z_{0} \in D_{n}$. Since $D-H$ is open in $D$, we can construct a path $\Gamma_{0}$ in $D-H$ that first joins $z_{0}$ to some point $z_{0}^{\prime} \in \sigma_{n}-H$ for some $\sigma_{n} \subset D$. Observe that there might be more than one path $\Gamma_{p / q}$ crossing the same arc $\sigma_{n}$. Let $\Gamma_{h} / m \subset H$ be the boundary path in $H$ with the property that $\Gamma_{h / m} \cap \sigma_{n}$ is the closest to $z_{0}^{\prime}$ on $\sigma_{n}$. From $z_{0}^{\prime}$, we make the path $\Gamma_{0} \subset D-H$ follows $\Gamma_{h / m}$ so closely in $D_{n}-H$ that $\Gamma_{0}$ intersects no path $\Gamma_{p / q} \subset H$, and so that the distance between $\Gamma_{0}$ and $\Gamma_{h / m}$ in the annulus $D_{n}$ approaches 0 as $n \rightarrow \infty$. Thus, $\Gamma_{0}$ connects $z_{0}$ to the boundary without intersecting $H$, and it ends at the point $e^{2 \pi i(h / m)}$ as desired.

\section{CONSTRUCTION OF THE FUNCTION $g$ ON $H$.}

$$
g(z)= \begin{cases}j & \text { for } z \in \gamma_{n} \subset C_{n}^{j}, j=0,1,2,3 \\ \frac{1}{1-|z|} & \text { for } z \in \Gamma_{p / q} \text { for all } p / q, q=1,2, \ldots ; p=1,2, \ldots, q-1 .\end{cases}
$$

Observe that $g \rightarrow \infty$ as $|z| \rightarrow 1$ along $\Gamma_{p / q}$. Note that $H$ has no interior and $g$ is continuous on $H$.

Construction of A FUnCtion $f \in \mathscr{A}-\mathscr{R}$. Since $g$ and $H$ satisfy the conditions of Lemma 1, there corresponds to every positive continuous function $\epsilon(t): 0<t<1$, some analytic function $f$ on $D$ with the property that

$$
|f(z)-g(z)|<\epsilon(d(z, C)) \quad \text { for all } z \in H .
$$


Let $f$ denote the function corresponding to $\epsilon_{0}(t)=1 / 9$ for all $t$.

Since

$$
g \rightarrow \infty \text { as }|z| \longrightarrow 1 \text { when } z \in \Gamma_{p / q}
$$

then

$$
f \longrightarrow \infty \text { as }|z| \longrightarrow 1 \text { when } z \in \Gamma_{p / q}
$$

as well, and since the paths $\Gamma_{p / q}$ end at a dense subset $S$ of $C$, it follows that $\infty$ is reached as an asymptotic value at a dense subset of the unit circle, that is $A_{\infty}$ is a dense subset of $C$, so that $f \in \mathscr{A}$, the MacLane class.

Since the function $g$ has values that differ by one on the arcs $\gamma_{n}$ of the different circles $C_{n}^{j}, j \in\{0,1,2,3\}$, the function $f$ has values that differ at least by $1-2(1 / 8)=3 / 4$ on the arcs $\gamma_{n}$ of the different circles $C_{n}^{j}, j \in\{0,1,2,3\}$. Therefore, if $n$ is sufficiently large, no asymptotic path can cross the arcs $\gamma_{n}$ on more than one of the four circles $C_{n}^{j}, j \in\{0,1,2,3\}$; hence, by a previous remark,

$$
\left|\Gamma_{p / q} \cap D_{n}\right| \geq \pi r_{n}^{0}\left(\frac{1}{n}-\frac{1}{2^{n}}\right) \text { for all } n>q .
$$

Finally, since the regions $D_{n}$ are disjoint and

$$
\Gamma=\cup_{n=1}^{\infty}\left\{\Gamma \cap D_{n}\right\},
$$

it follows that, for any asymptotic path $\Gamma$,

$$
|\Gamma| \geq \sum_{n} \pi r_{n}^{0}\left(\frac{1}{n}-\frac{1}{2^{n}}\right)=\infty .
$$

In other words, no asymptotic path is rectifiable, and so $f \notin \mathscr{R}$.

\section{REFERENCES}

[1] N. U. Arakeljan, Uniform and tangential approximations by analytic functions, Izv. Akad. Nauk Armjan. SSR Ser. Mat. 3 (1968), no. 4-5, 273-286 (Russian). MR 43\#530. Zbl 175.07602.

[2] E. F. Collingwood and A. J. Lohwater, The theory of cluster sets, Cambridge Tracts in Mathematics and Mathematical Physics, no. 56, Cambridge University Press, Cambridge, 1966. MR 38\#325. Zbl 149.03003.

[3] G. R. MacLane, Asymptotic values of holomorphic functions, Rice Univ. Studies 49 (1963), no. 1, 83. MR 26\#6419. Zbl 121.30203.

HAMDAN: LEBANESE AMERICAN UNIVERSITY, BEIRUT, LEBANON 


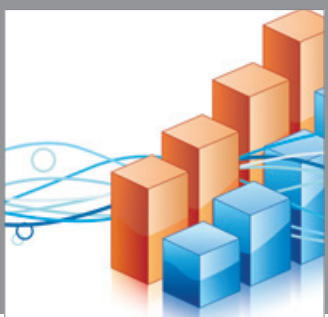

Advances in

Operations Research

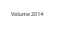

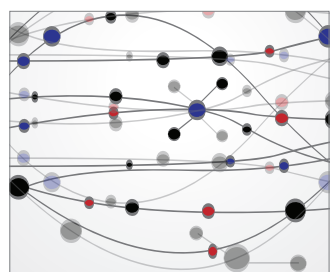

\section{The Scientific} World Journal
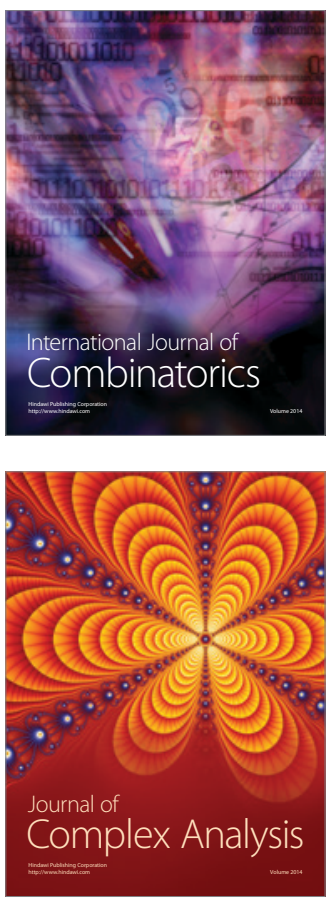

International Journal of

Mathematics and

Mathematical

Sciences
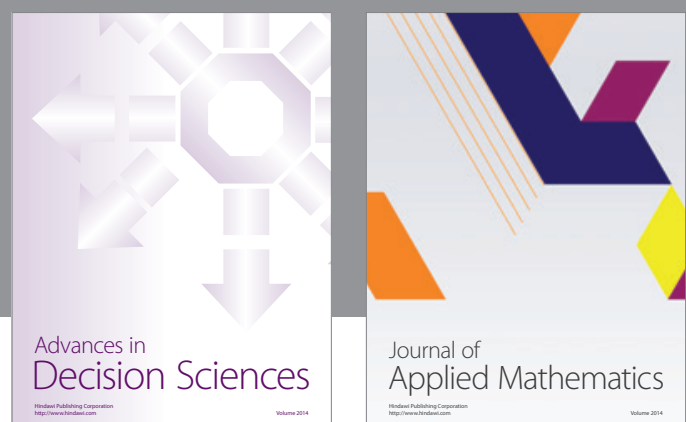

Journal of

Applied Mathematics
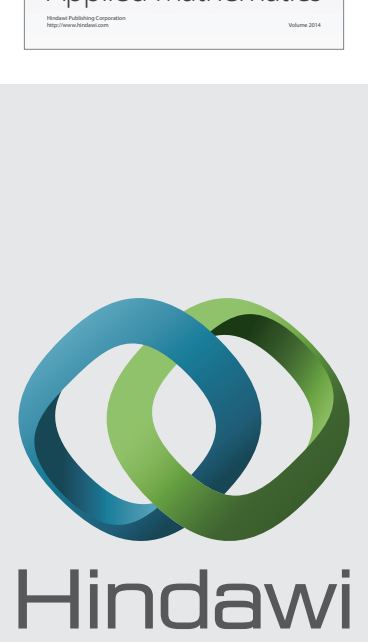

Submit your manuscripts at http://www.hindawi.com
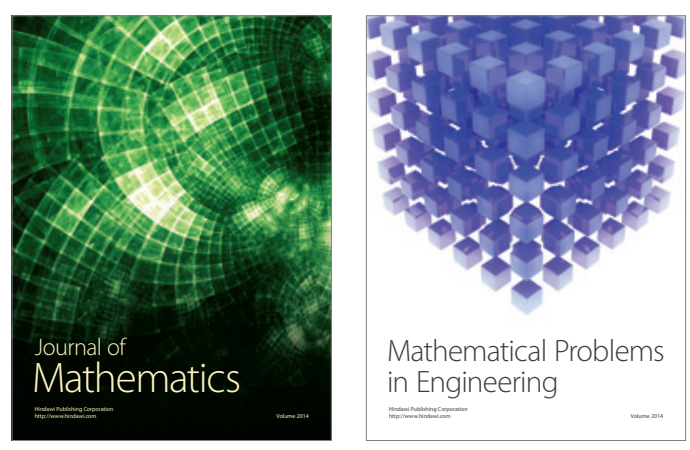

Mathematical Problems in Engineering
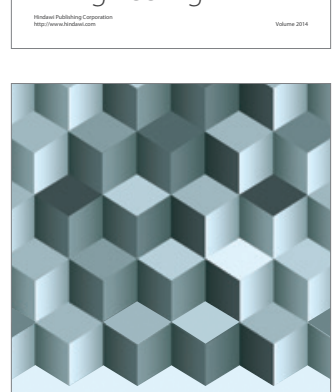

Journal of

Function Spaces
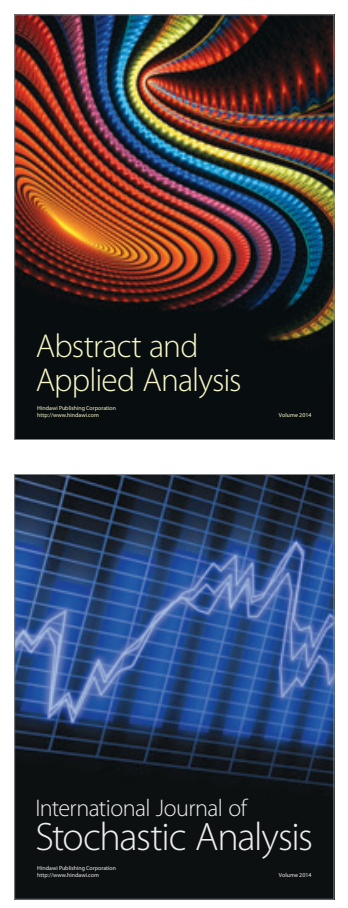

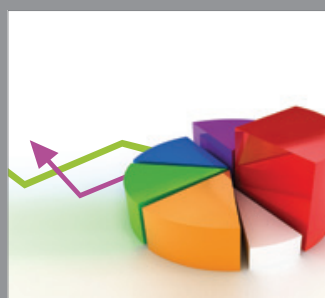

ournal of

Probability and Statistics

Promensencen
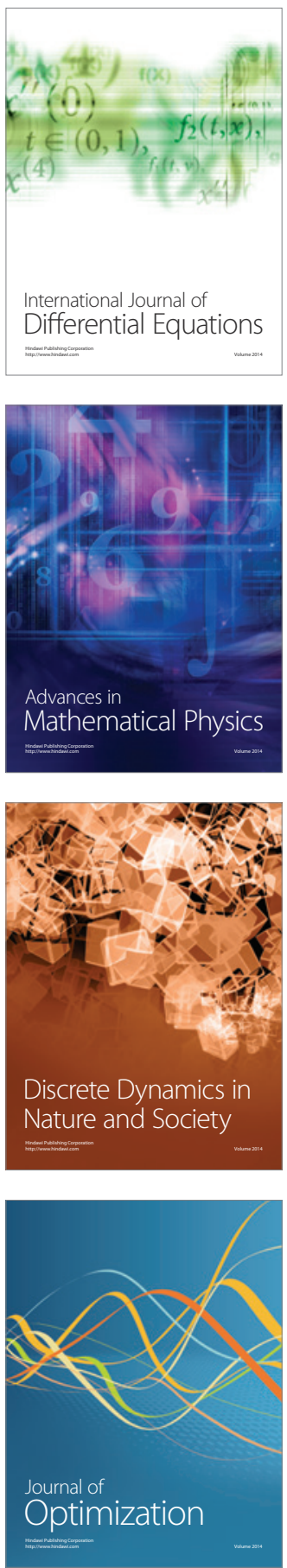